\title{
Aeronave de cuatro motores - drones USMP
}

Four - engine aircraft - drones

\author{
Recibido: julio 10 de 2015 | Revisado: agosto 21 de 2015 | Aceptado: octubre 02 de 2015
}

\section{Vihelmo Velapatiño ${ }^{1}$}

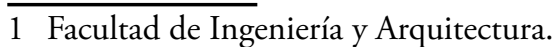
vvelapatinoc@usmp.pe

\begin{abstract}
The goal of the study was to develop a four-engine aircraft (drone) prototype for image acquisition, aerial video, freight of about 500 grams and 3D Laser object recognition system. The Quad-rotor methodology of design and construction was used, based on SURF navigation with which we were able to build the DRON-USMP prototype. This platform is equipped with sensors, autonomous navigation devices, GPS system and a security system of return to the point of takeoff when registering signal loss with the earth station. We also achieved flight experience and witnessed the proper development of the avionics on field tests on physical facilities of the Faculty of Engineering and Architecture at the University of San Martin de Porres, as well as in open fields in the district of La Molina.
\end{abstract}

Keywords: Quad-rotor drones, unmanned aircraft

\section{Resumen}

El objetivo del estudio fue desarrollar un prototipo de aeronave de cuatro motores (Dron) para la adquisición de imágenes, videos aéreos, transporte de carga de aproximadamente 500 gramos y con sistema de reconocimiento de objetos láser $3 \mathrm{D}$. Se usó la metodología de diseño y construcción del Quad-rotor, basado en la navegación SURF con lo cual se logró construir el prototipo DRON-USMP. Esta plataforma está equipada con sensores, dispositivos para navegación autónoma, sistema GPS y un sistema de seguridad de retorno al punto de despegue a la pérdida de señal con la estación terrena. Asimismo, se han experimentado vuelos y el desarrollo adecuado de la aviónica en pruebas de campo físicos en las instalaciones de la Facultad de Ingeniería y Arquitectura de la Universidad de San Martín de Porres y en campos abiertos del distrito de La Molina.

Palabras clave: Quad-rotor, drones, aeronaves no tripuladas 


\section{Introducción}

Los sistemas aéreos no tripulados, denominados hoy en día UAS por sus siglas en inglés "Unmanned Aircraft Systems" desarrollan misiones autónomas de vuelo controlados remotamente y la mayoría de los MICRO y MINI sistemas actuales han sido creados para la adquisición de imágenes y video con trayectorias de vuelo elementales como ascenso, descenso, izquierda, derecha, giro izquierda y derecha, controlados por un sistema remoto (Radio control RC) de tipo deportivo con rangos de vuelo menores a los $100 \mathrm{~m}$ de longitud que cumplen un mismo patrón único de diseño en las plataformas de tipos mixtos (Figura 1),es decir, simétricos en la rotación normal y coaxial de las hélices.

Según la Organización de Tratado del Atlántico Norte (OTAN), los UAS se clasifican en tres clases: CLASE I $=<150 \mathrm{~kg}$ de masa; CLASE II = de 150 hasta $600 \mathrm{KG}$ de masa; CLASE II $=>600 \mathrm{~kg}$ de masa. Con respecto a la CLASE I se subclasifica en tres tipos: MICRO $=<2 \mathrm{~kg}$ de masa y $5 \mathrm{~km}$ de rango de vuelo; MINI = de 2 hasta $20 \mathrm{~kg}$ de masa y $25 \mathrm{~km}$ de rango de vuelo y SMALL = > $20 \mathrm{~kg}$ de masa y $50 \mathrm{~km}$ de rango de vuelo. Estos tipos de robots correspondientes a la categoría MICRO han presentado diversos problemas en el cumplimiento de sus tareas asignadas tales como la adquisición de imágenes, las colisiones con estructuras, edificios, árboles, torres de alta tensión y red de cables; la unidad de suministro de energía y por lo tanto, la aplicación de sensores específicos para la medición y obtención de datos para la navegación óptima. A esto, se suman las restricciones legales que emite la FAA para la utilización de UAS y DRONES en lugares urbanos.

Esta investigación de la Escuela Profesional de Ciencias Aeronáuticas de la Facultad de Ingeniería y Arquitectura de la Universidad de San Martín de Porres presenta un prototipo MICRO UAS, diseñado y construido con el objetivo de transportar carga útil hasta 500 gr de masa (cámara de video o fotografía, caja de productos o medicina de $150 \mathrm{~mm}$ x $100 \mathrm{~mm}$ de longitud y de sensores Láser de reconocimiento para navegación anticolisión), suministrar y generar la transferencia de nuevas tecnologías en sistemas no tripulados para la utilización en los proyectos de investigación de la universidad.

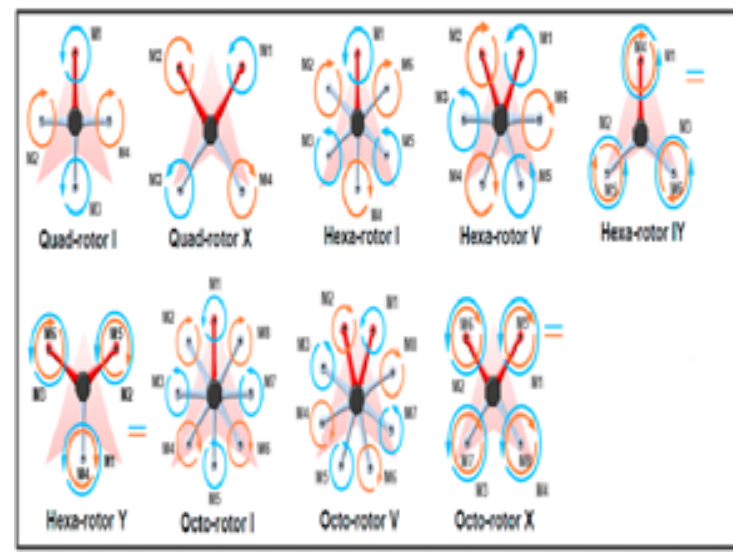

Figura 1. Diseño de plataformas de tipos mixtos y dirección de rotación de las hélices, Quad-rotor I y Quad-rotor X.

El Quad-rotor USMP es un prototipo de última generación (Figura 2) con plataforma construida con materiales de fibra de carbono, aluminio y plástico reforzado, la aviónica se constituye de Micro tarjeta de Control de Vuelo automático MC (Main controller), Módulo de navegación GPS/ Compass, Control de Velocidad de Motores ESC (Electronic Speed Controller), Unidad de Administración de Potencia PMU (Power Management Unit), receptor de RC y receptor Data Link para la gestión de vuelo en computador PC y/o Tablet para el control de orientación inteligente, además del sistema de propulsión (Motores Brushless y Hélices). El Quad-rotor está programado para aterrizar y retornar al punto de despegue inicial (Go-home and landing) en los siguientes casos: a) Cuando la batería alcanza un nivel de descarga mínima establecida y $b$ ) Cuando el Quad-rotor pierde contacto con la estación terrena (Naza-M 4.02). 


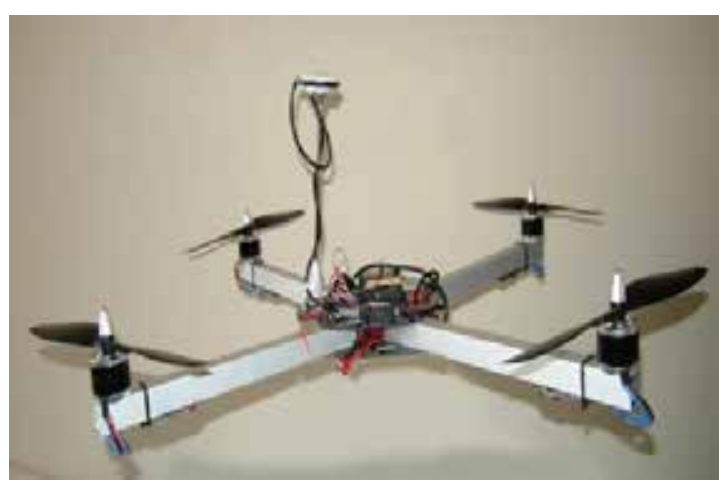

Figura 2. Quad-rotor USMP, vista lateral.

\section{Antecedentes}

Hasta la fecha, existe una amplia variedad de investigaciones que tratan de resolver el problema de anticolisión en vuelo en Quad-rotores para el transporte seguro de mercancías entre áreas urbanas. La existencia de edificios, estructuras, árboles e incluso personas impiden el normal desarrollo de vuelos de los UAS, para ello se han utilizado incluso cámaras de video, sensores de sonido, sensores ópticos, algoritmos de mapeo (Cummis \& Newman, 2011) basados en la navegación SURF (Bay, Ess, Tuytelaars, \& Van, 2008) con imágenes extraídas de cámaras omnidireccionales.

Para ello, en el presente prototipo, se incluyen terminales para la instalación de Scanners Láser Omnidireccionales (Tipaldi, Braun, \& Arreis, 2010) que resuelven adecuadamente el problema de navegación, en zonas urbanas con un alto índice de seguridad, frente a los obstáculos que se presenten.

\section{Método}

\section{Configuraciones del Quad-Rotor}

El dispositivo comprende cuatro hélices en configuración tipo " $\mathrm{X}$ ", de dos pares de hélices $(1,3)$ y $(2,4)$ que giran en direcciones opuestas por la variación de velocidad del rotor, se puede cambiar la fuerza de sustentación y crear movimiento, incrementando y disminuyendo la velocidad de las cuatro hélices, en simultáneo, que generan movimiento vertical. Cambiando la velocidad de las hélices 2 y 4 , se produce el movimiento lateral ROLL. La rotación PITCH es el resultado del cambio de velocidades de las hélices 1 y 3, y la rotación YAW es el resultado de los cambios de movimientos de los dos pares de hélices, respectivamente. La Figura 3 describe los movimientos del Quad-rotor de configuración tipo " $\mathrm{X}$ ”.

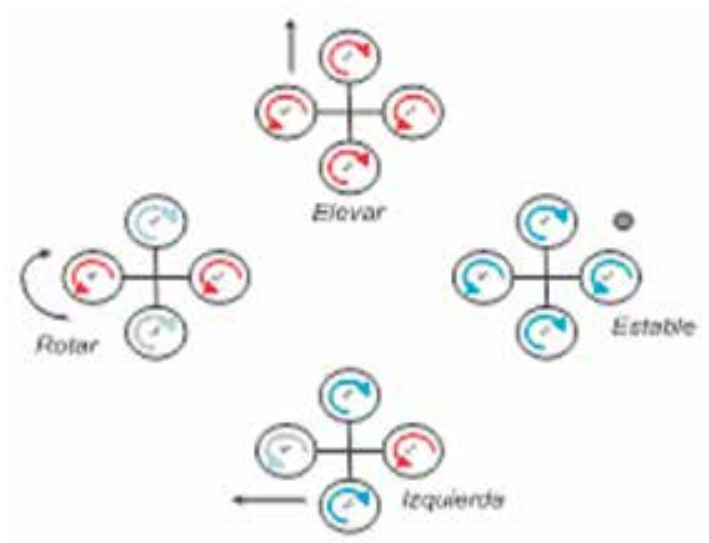

Figura. 3. Descripción de movimientos del Quad-rotor de configuración tipo " $X$ ".

\section{Resultados y discusión}

El Quad-rotor tiene cuatro rotores que son controlados independientemente. El movimiento del dispositivo es el resultado del cambio de velocidades de los rotores. La plataforma del Quad-rotor se considera rígida y simétrica, el centro de gravedad y la plataforma y del cuerpo de origen de la plataforma coinciden. Las hélices son rígidas y la fuerza de propulsión y la fuerza de resistencia son proporcionales al cuadrado de la velocidad de las hélices.

De acuerdo con estas consideraciones se establece la dinámica del sistema como un cuerpo rígido en el espacio y las fuerzas aerodinámicas causadas por el movimiento de los rotores. También se deben considerar los efectos giroscopios y otros que actúan en el Quad-rotor que se muestran en la Tabla 1 donde $C$ representan los términos constantes, $\omega$ es la velocidad del rotor, $J_{R}$ es el momento de inercia rotacional del rotor alrededor de su eje, $l$ es la distancia del centro de masa a los rotores, $J$ es el momento de 
inercia del cuerpo rígido y $\phi, \theta y \psi$ son los ángulos de Tait-Bryan.

Tabla 1

Principales efectos físicos que actúan sobre un Quad-rotor

\begin{tabular}{|c|c|c|}
\hline Efecto & Fuente & Formulación \\
\hline Efectos Acrodinámicos & $\begin{array}{l}\text { Rotación de Motores } \\
\text { Giro de hélices }\end{array}$ & $C \Omega^{2}$ \\
\hline Pares Inerciales Opuestos & $\begin{array}{l}\text { Cambio en la velocidad } \\
\text { de rotación de los rotores }\end{array}$ & $J_{n} \hat{\Omega}$ \\
\hline Efectos de la Gravedad & $\begin{array}{l}\text { Posición del centro de } \\
\text { masas }\end{array}$ & $t$ \\
\hline \multirow[t]{2}{*}{ Efectos Giroscópicos } & $\begin{array}{l}\text { Cambio en la orientación } \\
\text { del cuerpo rígido }\end{array}$ & $J \theta v$ \\
\hline & $\begin{array}{l}\text { Cambio en la orientación } \\
\text { del plano de los rotores }\end{array}$ & $J_{R} \Omega \theta, \phi$ \\
\hline Fricción & $\begin{array}{l}\text { Todos los movimientos } \\
\text { del belicóptero }\end{array}$ & $C \dot{\phi}, \dot{\theta}, \dot{v}$ \\
\hline
\end{tabular}

Nota: Bouabdallah, 2007.

Considerando un sistema de coordenada con orientación de mano-derecha (Figura 4), las tres rotaciones individuales son des- critas separadamente por:

- Alabeo (Roll), $\mathrm{R}(x, \phi)$, rotación alrededor del eje $x$.

- Cabeceo (Pitch), R ( $y, \theta)$, rotación alrededor del eje $y$.

- Guiñada (Yaw), R $(z, \psi)$, rotación alrededor del eje $z$

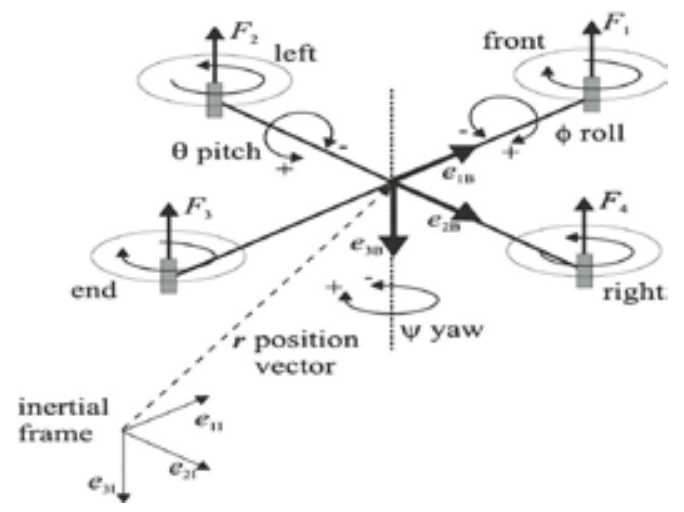

Figura 4. Estructura del Quad-rotor y los sistemas relativos de coordenadas.
El Quad-rotor está construido para recibir en conexión directa con el Laser Scanner Omnidireccional de $360^{\circ}$ para localización robot y mapeo (Robot localization $\&$ maping - SLAM). Para modelos 3D (Bovabdallah, 2007), seguridad y navegación anticolisión, rango de medición de $6 \mathrm{~m}$., sistema de visión con triangulación láser de alta velocidad y $7,9 \mathrm{~Hz}$ (474 RPM). Con el acoplamiento de este Scanner RPLIDAR (Figura 5), el Quad-rotor navega y con alta precisión, puede detectar y sortear obstáculos a una distancia de $6 \mathrm{~m}$., lo cual le facilita llegar al punto determinado sin colisiones ni accidentes.

La configuración general del Quad-rotor (Figura 6) facilita la incorporación de otros dispositivos electrónicos, cámaras de video y de fotografía y sensores hasta 500 gramos de masa.

\section{Conclusiones}

Se ha presentado un prototipo Quad-rotor que realiza vuelos autónomos con sistemas inteligentes de última generación. Asimismo, la utilización del Láser Scanner para modelos 3D facilita la navegación segura y adecuada del prototipo en trayectorias con obstáculos. El Quad-rotor USMP tiene numerosas aplicaciones: en el transporte de mercancías de hasta 500 gramos de masa (Delivery door to door), para la adquisición de imágenes y videos aéreos y utilización de sensores y monitoreo de zonas específicas (mineras, medio ambiente, seguridad urbana y otros). Nuestros primeros resultados y experiencias ayudaron a detectar nuevas tecnologías y aplicaciones del Quad-rotor que serán utilizados en nuestros futuros proyectos en la construcción de UAS. 


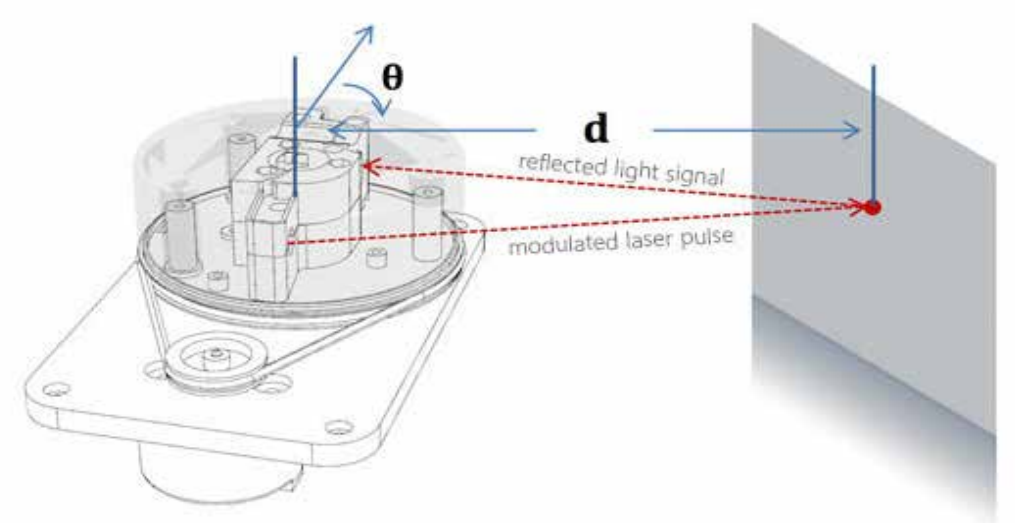

Figura 5. Omnidireccional Laser Scanner DSP con señal de laser infrarrojo modulado

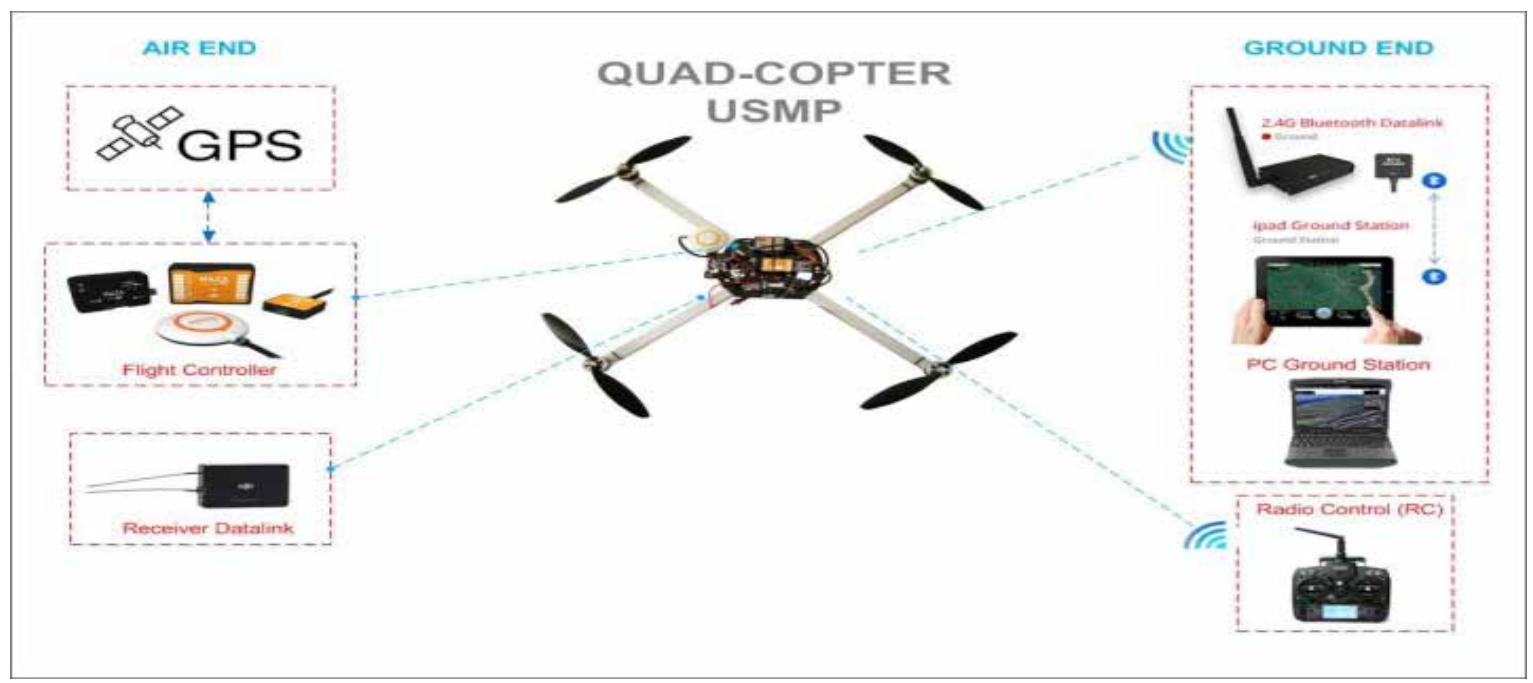

Figuras 6. Configuración General de Quad-rotor USMP

\section{Referencias}

Bay, H., Ess, A., Tuytelaars, T., \& Van, L. (junio, 2008). Speeded-Up Robust Features (Surf). Computer Vision and Image Understanding, 110(3), 346359.

Bouabdallah, S. (2007). Desing and control of quadrotors with application to autonomous flying (Tesis doctoral, École polytechnique fédérale de Lausanne, Switzerland).

Cummins, M. \& Newman, P. (2011) Appearance-only SLAM at large scale with FAB-MAP 2.0. The International Journal of Robotics Research, 30(9), 1100-1123.

Naza-M (Versión 4) [Controlador de vuelo]. Shenzhen, China: DJI.

Tipaldi, G., Braun, M., \& Arras, K. (2010). FLIRT: Interest regions for $2 D$ range data with applications to robot navigation. Trabajo presentado en International Symposium on Experimental Robotics (ISER), New Delhi, India. 
Vihelmo Velapatiño 